\title{
Modelación Matemática de Columnas de Destilación Alcohólica
}

\author{
Mathematical modeling of alcohol \\ distillation columns
}

\author{
Osney Pérez Ones ${ }^{1}$, Lourdes Zumalacárregui de Cárdenas², Osvaldo Gozá León ${ }^{3}$ \\ ${ }^{1}$ Facultad de Ingeniería Química, Instituto Superior Politécnico José Antonio Echeverría. Cuba, osney @qui imica. \\ cujae. edu.cu. \\ ${ }^{2}$ Facultad de Ingeniería Química, Instituto Superior Politécnico José Antonio Echeverría. Cuba, Iourdes@quimica . \\ cujae. edu.cu. \\ ${ }^{3}$ Facultad de Ingeniería Química, Instituto Superior Politécnico José Antonio Echeverría, ogoza@quimica. cujae. \\ edu.cu.
}

\section{Resumen}

Eneste trabajo se presenta una propuesta de módulos de cálculo paralaampliación del simuladormodular STA 4.0, orientado a la industria azucarera, con el objetivo de llevar a cabo el cálculo y análisis en destilerías de etanol. Se desarrollaron los módulos de cálculo para la simulación de las columnas que conforman el área de destilación, teniendo en cuenta los balances de materiales y energía, las relaciones de equilibrio y las propiedades termodinámicas del sistema etanol-agua. Para la determinación de las etapas teóricas en las columnas se hace uso del método de Ponchon-Savarit. Para la columna destiladora, se estableció una comparación entre los resultados obtenidos con los que ofrece el método de McCabe-Thiele. Se ilustra la aplicación de estos módulos de cálculo de destilerías de etanol a un caso real, demostrando su validez.

Palabras clave: modelación, etanol, destilación

\section{Abstract}

New evaluation modules are proposed to extend the scope of a modular simulator oriented to the sugar cane industry, called STA 4.0, in a way that it can be used to carry out $x$ calculation and analysis in ethanol distilleries. Calculation modules were developed for the simulation of the columns that are combined in the distillation area. Mathematical models were supported on materials and energy balances, equilibrium relations and thermodynamic properties of the ethanol-water system. Ponchon-Savarit method was used for the evaluation of the theoretical stages in the columns. A comparison between the results using Ponchon- Savarit method and those obtained applying McCabe-Thiele method was done for a distillation column. These calculation modules for ethanol distilleries were applied to a real case for validation.

Keywords: modeling, ethanol, distillation

Recibido: 1/05/2011

Aceptado: 23/06/2011

\section{Introducción}

El proceso de producción de etanol ha sido inmensamente refinado y actualizado en años recientes lo que le ha permitido mayor eficacia. Este varía ligeramente para cada uno de los tres usos principales del etanol (bebidas, industrial y combustible), pero los pasos fundamentales para su obtención son los mismos [1]. La fermentación es el primer paso para la obtención del etanol, proceso donde los azúcares contenidos en los jugos de caña y las mieles son transformados con la ayuda de levaduras. Después, el etanol fermentado pasa a las columnas de destilación, dondes a través de un proceso de evaporación los 
compuestos se separan y se obtiene el alcohol y la vinaza. La etapa final es la deshidratación, en la que se retira el agua del alcohol y se obtiene el etanol carburante o el etanol anhidro. La producción del etanol es considerada por algunos estudiosos como una gran alternativa para el cambio de la matriz energética, por ser clasificada como una fuente limpia y renovable de energía. [2]. Actualmente, el problema de la energía no es solo un problema de las industrias químicas, sino un problema mundial. La humanidad se enfrenta ante la problemática de una demanda energética cada vez más creciente junto con unas fuentes energéticas convencionales cada vez más escasas. La mayor parte del uso energético causa daño al medio ambiente. La quema de combustibles fósiles es la causa principal de las emisiones de dióxido de carbono, el más importante de los gases de efecto invernadero, lo que ocasiona las lluvias ácidas y la contaminación atmosférica.

Algunos estudios sugieren que los países más desarrollados pueden reducir su consumo energético, y, al mismo tiempo, mantener el ritmo de crecimiento, empleando las fuentes renovables de energía. [3], [4]. El ahorro, es una práctica empleada durante el consumo de energía, que tiene como objetivo disminuir su uso, pero con el mismo resultado final. Esta práctica conlleva un aumento del capital financiero, ambiental, de la seguridad nacional, de la seguridad personal y del confort humano. Una vía rápida y económica para obtener información de una industria, ya sea desde el punto de vista energético o de proceso, es el uso de simuladores. El concepto de simulación ha sido planteado por Scenna como: "La simulación de un proceso consiste en la resolución por computadora de un modelo matemático que describe su comportamiento a través de variables que determinan su estado" [14].

La simulación de procesos ha encontrado su mayor aplicación con el uso de los simuladores comerciales en industrias como la petroquímica y la del papel, pero también existen aplicaciones en la industria azucarera y la alcoholera, especialmente con el objetivo de buscar mayores eficiencias energéticas. En el Instituto Superior Politécnico "José Antonio Echeverría" de la Habana -Cuba, se dispone del Simulador STA 4.0, orientado a la simulación de sistemas termoenergéticos de fábricas de azúcar crudo y refino [5]. Los modelos matemáticos hasta el momento desarrollados en este simulador, no permiten abordar la simulación de destilerías de etanol en toda su amplitud y rigor. De lo anterior se infiere, que es necesario realizar una ampliación del STA 4.0, mediante la incorporación de nuevos módulos de cálculo, que le permitan obtener una mayor cantidad de información para el análisis de los sistemas termoenergéticos en fábricas de etanol [6].

\section{Contenido}

\section{Descripción de los modelos matemáticos}

Para la simulación del área de destilación de etanol se requiere módulos de cálculo que representen los diferentes tipos de columnas que pueden utilizarse en el proceso. Estas son: destiladora, destiladora con rehervidor, depuradora, despojadora, rectificadora y deshidratadora. Se desarrollaron los módulos de cálculo para cada columna, los cuales fueron incorporados al simulador STA 4.0. En la Figura 1 se presentan los esquemas de estas columnas. Para el desarrollo de cada módulo se requieren dos fases de cálculo una primera en la que se determinan las condiciones de entrada y salida de cada corriente en la columna, siguiendo la metodología de Rudd y Watson [7]; en la segunda fase se determina la cantidad de platos teóricos en el equipo. En este trabajo se desarrolla la metodología aplicada presentado el resultado de la simulación de la columna destiladora con y sin rehervidor, así como una comparación entre los dos métodos clásicos de cálculo de platos teóricos, McCabe-Thiele y Ponchon-Savari [8]. 

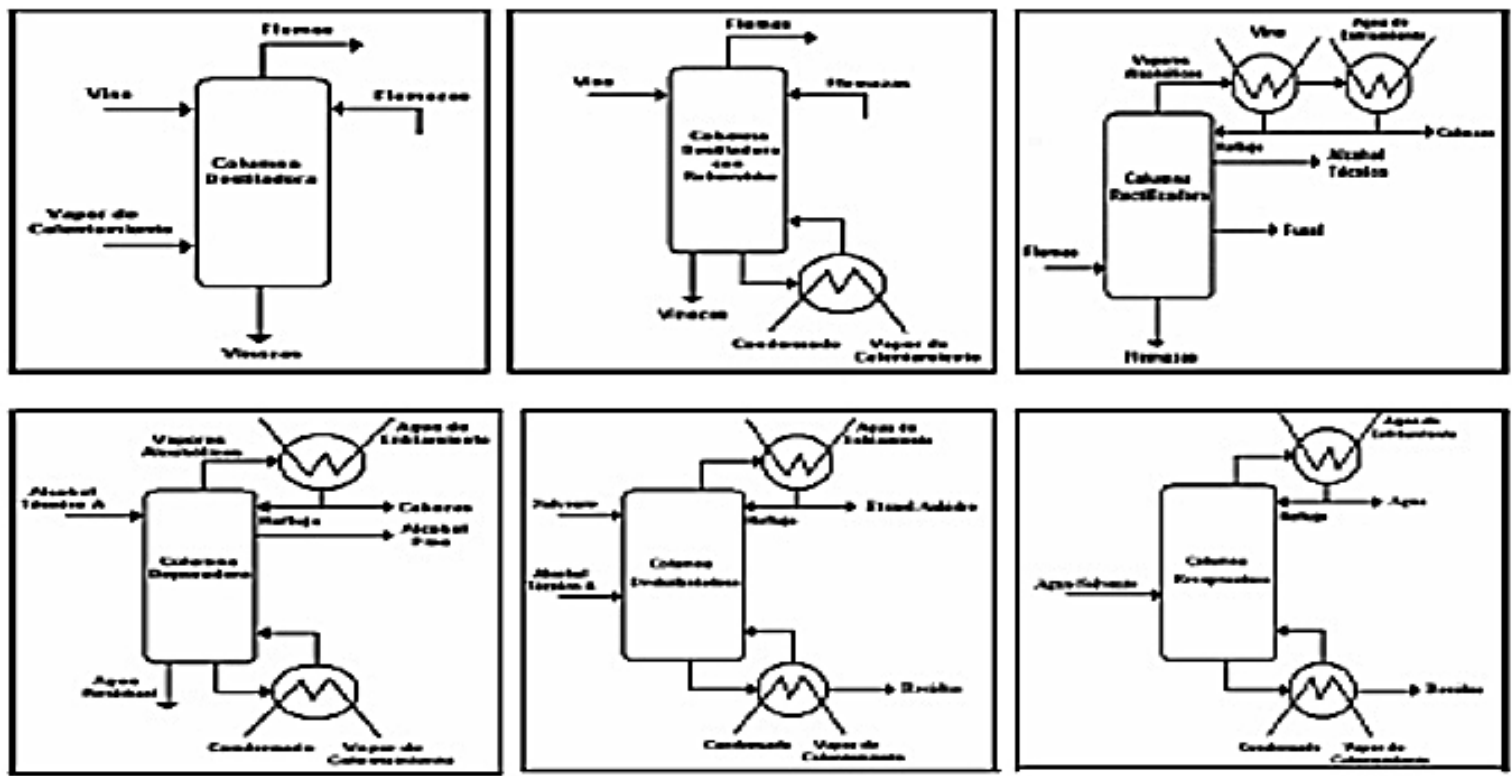

Fuente: [8].

Figura 1. Módulos de cálculos desarrollados

\section{Módulo columna destiladora}

Una columna destiladora se utiliza para elimina la mayor parte del agua vino junto con los sólidos suspendidos, sales minerales disueltas y otros productos no volátiles

\section{Balance de materiales y energía en la columna}

Objetivos de este módulo:

- Determinar el consumo de vapor de calentamiento y la eficiencia de separación.

- Determinar los platos teóricos y la eficiencia global de la columna, así como la temperatura y las composiciones del líquido y del vapor para cada uno de los platos teóricos.

Suposiciones del modelo:

- Operación continua isobárica y adiabática.

- Mezcla binaria etanol (1) agua (2).

- Equilibrio termodinámico entre la fase líquida y vapor que sale de cada plato.

- La alimentación es líquido saturado o líquido subenfriado, mientras que la recirculación es líquido saturado.

5. Todos los sólidos que entran a la columna salen en las vinazas.

En este módulo de cálculo se pueden señalar dos casos fundamentales: con y sin recirculación.

\section{Columna destiladora sin recirculación}

En la figura 2 se presenta el modelo para el caso en que la columna no presente recirculación. 


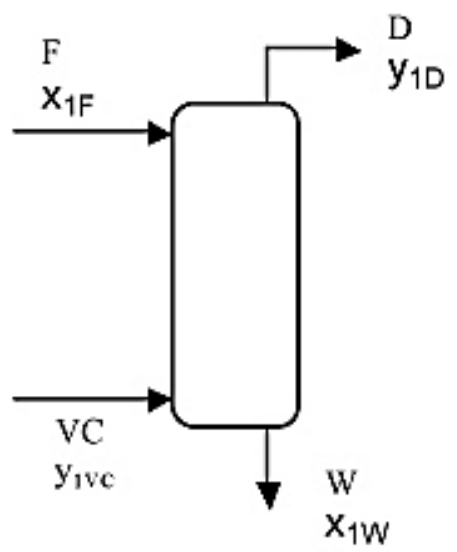

Figura 2. Columna destiladora sin recirculación

Balance global

$$
\begin{aligned}
& F+V C=D+W \\
& V C=D+W-F
\end{aligned}
$$

Balance por componente

$x_{1 F} F=y_{1 D} D+x_{1 W} W$

$W=\frac{x_{1 F} F-y_{1 D} D}{x_{1 W}}$

Balance de energía

$W h_{W}+D h_{D}-F h_{F}-V C h_{V C}=0$

$E S=\frac{D y_{1 D}}{F x_{1 F}} * 100$

$h_{F}=f\left(x_{1 F} ; T_{F}\right)$

$h_{W}=f\left(x_{1 W} ; T_{W}\right)$ 
$h_{D}=f\left(y_{1 D} ; T_{D}\right)$

$h_{V C}=f\left(P_{V C}\right)$

Cantidad de ecuaciones linealmente independientes: 8.

Cantidad de variables totales: 16 .

Grados de libertad: 8.

A partir de los grados de libertad del sistema se seleccionan las variables de decisión y las variables por calcular.

Variables de decisión

1. F: Flujo de vino $(\mathrm{kg} / \mathrm{h})$.

2. $x_{1 F}$ : Fracción másica de etanol en vino.

3. $T_{F}$ : Temperatura del vino $\left({ }^{\circ} \mathrm{C}\right)$.

4. $x_{1 W}$ : Fracción másica de etanol en vinazas (adim)

5. $T_{w}$ : Temperatura de las vinazas $\left({ }^{\circ} \mathrm{C}\right)$.

6. $\quad P_{v c}:$ Presión del vapor $(\mathrm{Pa})$.

7. $y_{10}$ : Fracción másica de etanol en flemas (adim).

8. $T_{D}$ : Temperatura de las flemas $\left({ }^{\circ} \mathrm{C}\right)$.

Variables calculables

1. $h_{F}$ : Entalpía de la corriente de vino $(\mathrm{kJ} / \mathrm{kg})$.

2. $h_{w}$ : Entalpía de la corriente de vinazas $(\mathrm{kJ} / \mathrm{kg})$.

3. $h_{D}$ : Entalpía de la corriente de flemas $(\mathrm{kJ} / \mathrm{kg})$.

4. $h_{v c}$ : Entalpía de la corriente de vapor $(\mathrm{kJ} / \mathrm{kg})$.

5. ES: Eficiencia de separación (\%).

6. D: Flujo de flemas $(\mathrm{kg} / \mathrm{h})$.

7. VC: Flujo de vapor $(\mathrm{kg} / \mathrm{h})$.

8. W: Flujo de vinazas $(\mathrm{kg} / \mathrm{h})$.

Aplicando la metodología de Rudd y Watson [7], se determina la secuencia de cálculos para la solución del sistema de ecuaciones. En la Tabla 1, se presenta esta secuencia para la columna destiladora sin recirculación.

Tabla 1. Secuencia de cálculos para el sistema columna destiladora sin recirculación

\begin{tabular}{|c|c|c|c|c|c|c|c|c|}
\hline \hline Orden de cálculo & 1 & 1 & 1 & 1 & 2 & 3 & 4 & 4 \\
\hline Variable a determinar & $\mathrm{h}_{\mathrm{F}}$ & $\mathrm{h}_{\mathrm{W}}$ & $\mathrm{h}_{\mathrm{D}}$ & $\mathrm{h}_{\mathrm{vC}}$ & $\mathrm{D}$ & $\mathrm{W}$ & $\mathrm{ES}$ & $\mathrm{VC}$ \\
\hline Ecuación a utilizar & 5 & 6 & 7 & 8 & 3 & 2 & 4 & 1 \\
\hline
\end{tabular}




\section{Columna destiladora con recirculación}

En la figura 3 se presenta el modelo para el caso en que la columna tenga recirculación.

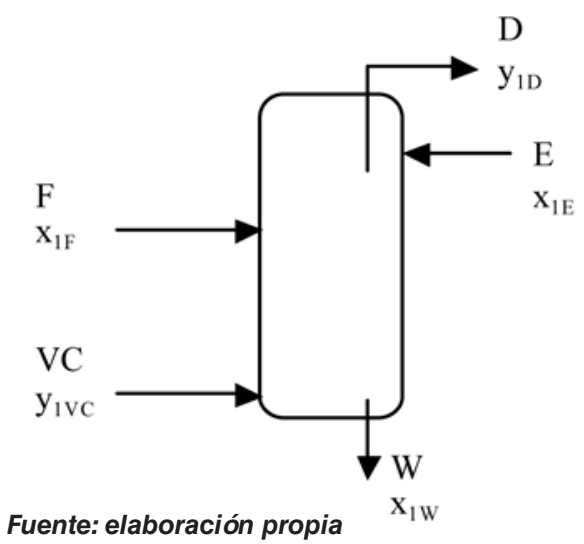

Figura 3. Columna destiladora con recirculación

Balance global

$$
\begin{aligned}
& F+V C+E=W+D \\
& V C=W+D-F-E
\end{aligned}
$$

Balance por componente.

$$
\begin{aligned}
& F x_{1 F}+E x_{1 E}=W x_{1 W}+D y_{1 D} \\
& W=\frac{F x_{1 F}+E x_{1 E}-D y_{1 D}}{x_{1 W}}
\end{aligned}
$$

Balance de energía.

$$
\begin{aligned}
& W h_{W}+D h_{D}-F h_{F}-V C h_{V C}-E h_{E}=0 \\
& \left(\frac{F x_{1 F}+E x_{1 E}-D y_{1 D}}{x_{1 W}}\right) h_{w}+D h_{D}-F h_{F}-\left[\left(\frac{F x_{1 F}+E x_{1 E}-D y_{1 D}}{x_{1 W}}\right)+D-F-E\right] h_{V C}-E h_{E}=0 \\
& E S=\frac{D y_{1 D}}{F x_{1 F}+E x_{1 E}} * 100
\end{aligned}
$$


$h_{F}=f\left(x_{I F} ; T_{F}\right)$

$h_{W}=f\left(x_{1 W} ; T_{W}\right)$

$h_{D}=f\left(y_{1 D} ; T_{D}\right)$

$h_{E}=f\left(x_{1 E} ; T_{E}\right)$

$h_{V C}=f\left(P_{V C}\right)$

Ecuaciones linealmente independientes: 9.

Cantidad de variables totales: 20 .

Grados de libertad: 11.

Variables de decisión:

1. F: Flujo de vino $(\mathrm{kg} / \mathrm{h})$.

2. $x_{1 F}$ : Fracción másica de etanol en vino (adim).

3. $T_{F}$ :Temperatura del vino $\left({ }^{\circ} \mathrm{C}\right)$.

4. $x_{1 W^{*}}$ :Fracción másica de etanol en vinazas (adim)

5. $T_{w}$ :Temperatura de las vinazas $\left({ }^{\circ} \mathrm{C}\right)$.

6. $\quad P_{v c}$ : Presión del vapor $(\mathrm{Pa})$.

7. $y_{1 D}$ : Fracción másica de etanol en flemas (adim).

8. $T_{D}$ : Temperatura de las flemas $\left({ }^{\circ} \mathrm{C}\right)$.

9. $\mathbf{x}_{1 \mathrm{E}}$ : Fracción másica de etanol en flemazas (adim).

10. $T_{E}$ :Temperatura de las flemazas $\left({ }^{\circ} \mathrm{C}\right)$.

11. E: Flujo de flemazas $(\mathrm{kg} / \mathrm{h})$.

Variables calculables:

1. $h_{F}$ : Entalpía de la corriente de vino $(\mathrm{kJ} / \mathrm{kg})$.

2. $h_{w}$ : Entalpía de la corriente de vinazas $(\mathrm{kJ} / \mathrm{kg})$.

3. $h_{D^{0}}$ : Entalpía de la corriente de flemas $(\mathrm{kJ} / \mathrm{kg})$.

4. $h_{v c}$ : Entalpía de la corriente de vapor $(\mathrm{kJ} / \mathrm{kg})$.

5. $h_{E}$ : Entalpía de la corriente de flemazas $(\mathrm{kJ} / \mathrm{kg})$.

6. ES: Eficiencia de separación (\%). 
7. D: Flujo de flemas $(\mathrm{kg} / \mathrm{h})$.

8. VC: Flujo de vapor $(\mathrm{kg} / \mathrm{h})$.

9. W: Flujo de vinazas $(\mathrm{kg} / \mathrm{h})$.

Al aplicar la metodología de Rudd y Watson (1987) se determina la secuencia de cálculos para la solución del sistema de ecuaciones. En la Tabla 2 se presenta esta para la columna destiladora con recirculación.

Tabla 2. Secuencia de cálculos para el sistema columna destiladora con recirculación

\begin{tabular}{||c|c|c|c|c|c|c|c|c|c||}
\hline \hline Orden de cálculo & 1 & 1 & 1 & 1 & 2 & 3 & 4 & 5 & 5 \\
\hline Variable a determinar & $\mathrm{h}_{\mathrm{F}}$ & $\mathrm{h}_{\mathrm{W}}$ & $\mathrm{h}_{\mathrm{D}}$ & $\mathrm{h}_{\mathrm{E}}$ & $\mathrm{h}_{\mathrm{VC}}$ & $\mathrm{D}$ & $\mathrm{W}$ & $\mathrm{ES}$ & $\mathrm{VC}$ \\
\hline Ecuación a utilizar & 5 & 6 & 7 & 8 & 9 & 3 & 2 & 4 & 1 \\
\hline
\end{tabular}

Fuente: elaboración propia

\section{Módulo columna destiladora con rehervidor}

\section{Fase 1: Balance de materiales y energía en la columna}

Los objetivos y suposiciones son iguales a los del módulo de la columna destiladora. Se presenta el caso en que la columna opera con un rehervidor total y con recirculación de flemazas, como se presenta en la Figura 4.

1.2.1 Columna destiladora con rehervidor con recirculación

Balance global

$$
\begin{aligned}
& F+E=D+W \\
& W=F+E-D
\end{aligned}
$$

Balance por componente

$$
\begin{aligned}
& F x_{1 F}+E x_{1 E}=D y_{1 D}+W x_{1 W} \\
& F x_{1 F}+E x_{1 E}=D y_{1 D}+(F+E-D) x_{1 W}
\end{aligned}
$$

Balance de energía

$$
\begin{aligned}
& (F+E-D) h_{W}+D h_{D}-F h_{F}-E h_{E}=\left(\frac{\% P}{100}-1\right) \cdot\left[V C\left(h_{V C S}-h_{V C E}\right)\right] \\
& E S=\frac{D y_{1 D}}{F x_{1 F}+E x_{1 E}} \cdot 100
\end{aligned}
$$


$h_{W}=f\left(T_{W} ; x_{1 W}\right)$

$h_{D}=f\left(T_{D} ; y_{1 D}\right)$

$h_{F}=f\left(T_{F} ; x_{1 F}\right)$

$h_{E}=f\left(T_{E} ; x_{1 E}\right)$

$h_{V C S}=f\left(P_{V C} ;\right.$ estado. fisico-técnico $)$

$h_{V C E}=f\left(P_{V C} ;\right.$ estado. fisico-técnico $)$

Ecuaciones linealmente independientes: 19

Cantidad de variables totales: 23

Grados de libertad: 13

Variables de decisión:

1. $F$ : Flujo de la corriente de vino $(\mathrm{kg} / \mathrm{h})$.

2. $x_{1 F}$ : Fracción másica de etanol en la corriente de vino vino (adim.).

3. $T_{F}$ : Temperatura de la corriente de vino $\left({ }^{\circ} \mathrm{C}\right)$.

4. $y_{1 D}$ : Fracción másica de etanol en la corriente de flemas (adim.).

5. $T_{D}$ : Temperatura de la corriente de flemas $\left({ }^{\circ} \mathrm{C}\right)$.

6. $E$ : Flujo de la corriente de flemazas $(\mathrm{kg} / \mathrm{h})$.

7. $x_{1 E}$ : Fracción másico de etanol en la corriente de flemazas (adim.).

8. $T_{E}$ : Temperatura de la corriente de flemazas $\left({ }^{\circ} \mathrm{C}\right)$.

9. $x_{1 W}$ : Fracción másico de etanol en la corriente de vinazas (adim.).

10. $T_{W}$ : Temperatura de la corriente de vinazas $\left({ }^{\circ} \mathrm{C}\right)$.

11. $P_{\mathbb{K}}$ : Presión del vapor de calentamiento $(\mathrm{kPa})$.

12. $\% P$ : Por ciento de pérdidas de calor en el rehervidor (\%).

13. Estado físico-técnico del vapor de calentamiento. 
Variables calculables:

1. $D$ : Flujo de la corriente de flemas $(\mathrm{kg} / \mathrm{h})$.

2. $W$ : Flujo de la corriente de vinazas $(\mathrm{kg} / \mathrm{h})$.

3. $h_{W}$ : Entalpía de la corriente de vinazas $(\mathrm{kJ} / \mathrm{kg})$.

4. $h_{D}$ : Entalpía de la corriente de flemas $(\mathrm{kJ} / \mathrm{kg})$.

5. $h_{F}$ : Entalpía de la corriente de vino $(\mathrm{kJ} / \mathrm{kg})$.

6. $h_{E}$ : Entalpía de la corriente de flemazas $(\mathrm{kJ} / \mathrm{kg})$.

7. $\quad$ : Flujo del vapor de calentamiento $(\mathrm{kg} / \mathrm{h})$.

8. $h_{V C S}$ : Entalpía de la corriente de salida del vapor de calentamiento $(\mathrm{kJ} / \mathrm{kg})$.

9. $h_{V C E}$ : Entalpía de la corriente de entrada del vapor de calentamiento $(\mathrm{kJ} / \mathrm{kg})$.

10. $\boldsymbol{B}$ : Eficiencia de separación (\%).

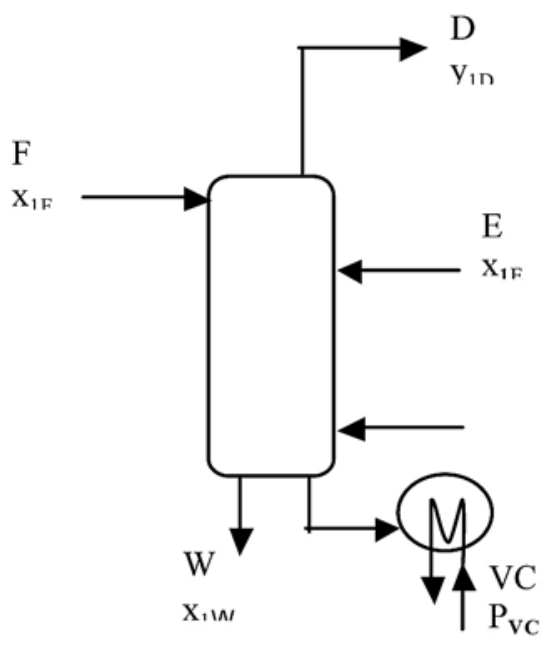

Fuente: elaboración propia

Figura 4. Columna destiladora con rehervidor con recirculación

Tabla 3. Secuencia de cálculos para el sistema columna destiladora con rehervidor con recirculación

\begin{tabular}{|c|c|c|c|c|c|c|c|c|c|c||}
\hline \hline Orden de cálculo & 1 & 1 & 1 & 1 & 1 & 1 & 1 & 2 & 3 & 3 \\
\hline Variable a determinar & $\mathrm{h}_{\mathrm{F}}$ & $\mathrm{h}_{\mathrm{W}}$ & $\mathrm{h}_{\mathrm{D}}$ & $\mathrm{h}_{\mathrm{E}}$ & $\mathrm{h}_{\mathrm{VCE}}$ & $\mathrm{h}_{\mathrm{VCS}}$ & $\mathrm{D}$ & $\mathrm{VC}$ & $\mathrm{W}$ & $\mathrm{ES}$ \\
\hline Ecuación a utilizar & 5 & 6 & 7 & 8 & 9 & 10 & 2 & 3 & 1 & 4 \\
\hline
\end{tabular}

Fuente: elaboración propia

\section{Fase 2: Algoritmo de conteo de platos teóricos}

Se continúa el módulo con el conteo de platos teóricos (NE) necesarios para lograr la separación deseada. Para ello, se utilizan por lo general dos métodos gráficos: el método de Ponchon-Savarit [8] llamado también concentración-entalpía, que es más riguroso, ya que requiere datos detallados de entalpía para poderse aplicar. El segundo, es el método de McCabe-Thiele [8] el cual es menos exacto que el anterior. Esto es debido a que supone que por cada mol que se evapora del componente más volátil se condensa un mol del componente menos volátil, además solo utiliza las relaciones de equilibrio y el balance de materiales en su aplicación. 
Los métodos gráficos consumen mucho tiempo y son ciertamente inexactos. Por otro lado, es posible hacer dichos cálculos utilizando alguno de los programas comerciales para la simulación de procesos químicos [9]. Para el caso de la columna destiladora con y sin recirculación, se utilizó el algoritmo del conteo de etapas (desde el fondo hacia el tope de la torre) y se utilizaron ambos métodos, se determinó para cada plato la composición del líquido y del vapor en equilibrio, así como la temperatura. Los algoritmos obtenidos por el método de McCabe-Thiele y por el método de Ponchon-Savarit se muestran en las figuras 5 y 6 respectivamente. El resto de los modelos fueron desarrollados de igual forma.

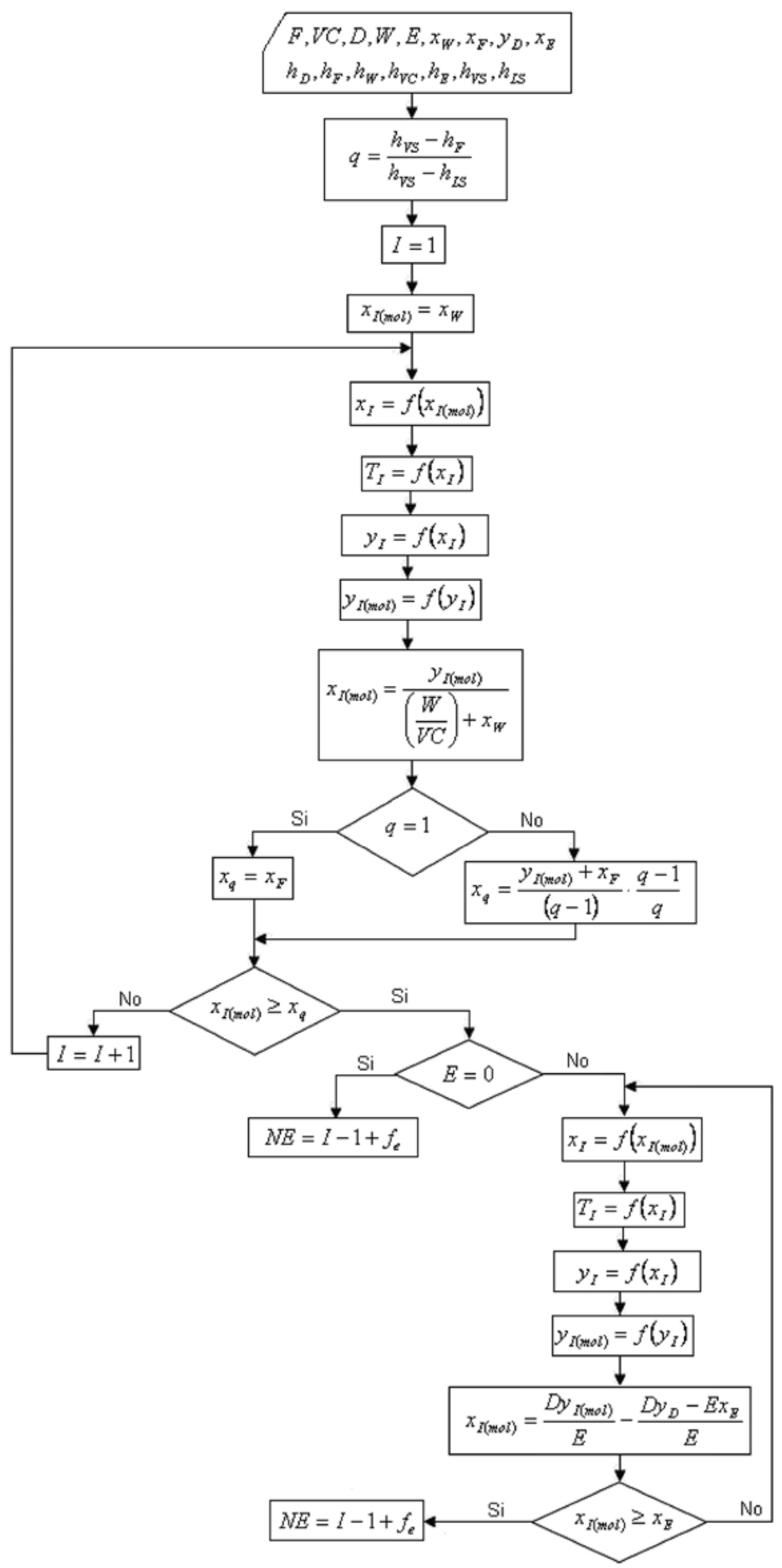

Figura 5. Algoritmo de conteo de etapas teóricas por el método de McCabe-Thiele 


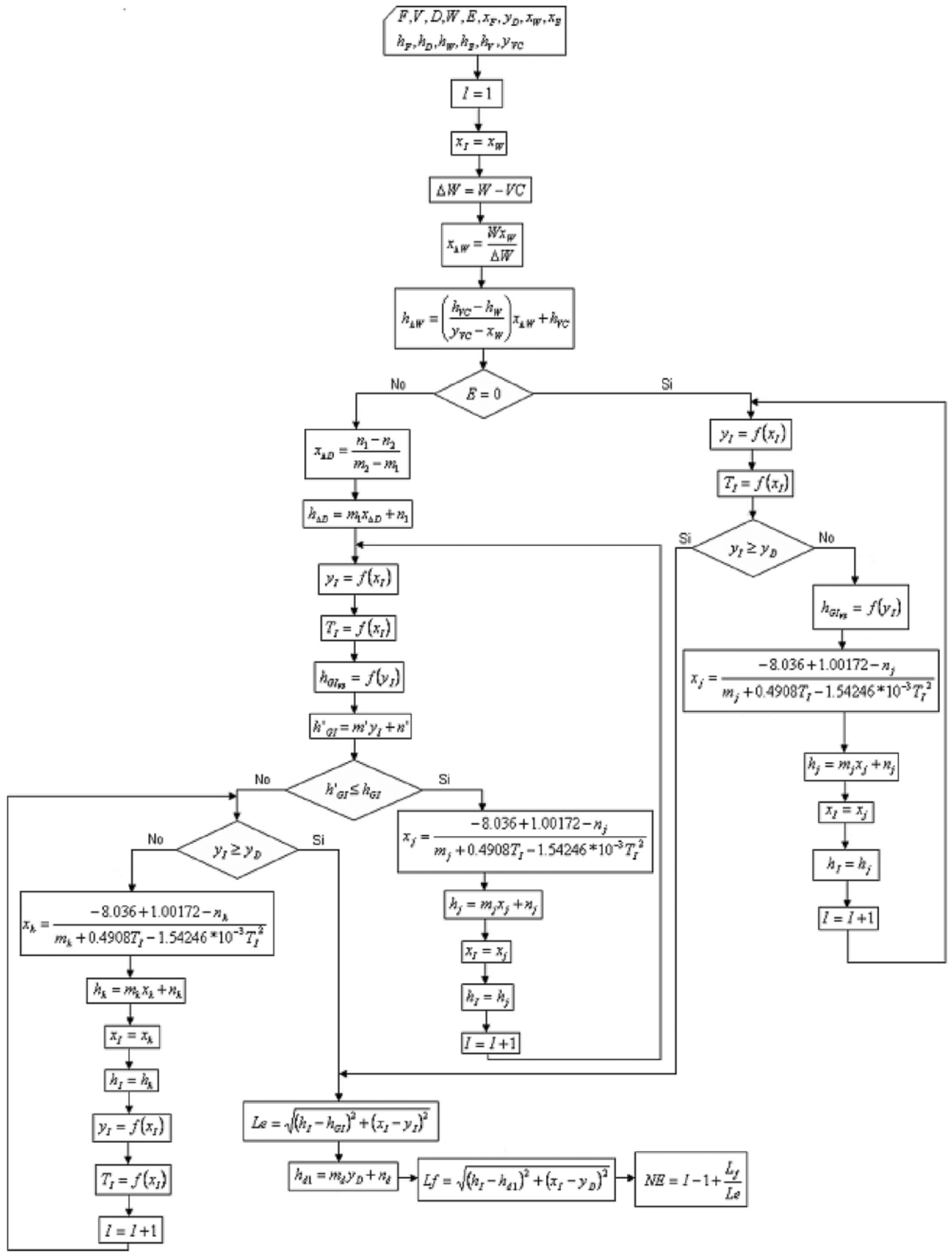

Figura 6. Algoritmo de conteo de etapas teóricas por el método de Ponchon-Savarit 


\section{Resultados y Discusión}

Para conocer la confiabilidad de los módulos de cálculo propuestos, se realizó la programación de las ecuaciones de los balances de materiales y energía en una hoja Excel®. Las propiedades físicas y termodinámicas de cada una de las corrientes se calcularon mediante modelos matemáticos desarrollados anteriormente y que están incorporados en el simulador. El resto de las variables de decisión introducidas provienen de un caso real de operación en una destilería de la provincia Mayabeque, Cuba. En esta, opera una columna destiladora a presión atmosférica, de 19 platos, que recibe $43.745 \mathrm{~kg} / \mathrm{h}$ de vino a $60^{\circ} \mathrm{C}$ con una fracción másica de etanol de 0,041.

El vapor de calentamiento utilizado es saturado a $136 \mathrm{kPa}$. Se validaron los siguientes módulos: columna destiladora con y sin recirculación, columna destiladora con rehervidor con y sin recirculación, columna rectificadora y columna depuradora. En las tablas 4,5 y 6 se presentan los resultados de las variables calculadas para la columna destiladora sin y con recirculación y la destiladora con rehervidor y recirculación.

Tabla 4. Resultados de las variables calculables para la columna destiladora sin recirculación

\begin{tabular}{||l|c|c||}
\hline \multicolumn{3}{|c||}{ VARIABLES CALCULABLES } \\
\hline \multicolumn{1}{|c|}{ Variable } & Valor & Unidades \\
\hline Flujo de flemas & 5661,1 & $\mathrm{~kg} / \mathrm{h}$ \\
\hline Flujo de vinazas & 45841,2 & $\mathrm{~kg} / \mathrm{h}$ \\
\hline Flujo del vapor de calentamiento & 7716,3 & $\mathrm{~kg} / \mathrm{h}$ \\
\hline Eficiencia de separación & 92,1 & $\%$ \\
\hline
\end{tabular}

Fuente: elaboración propia

Tabla 5. Resultados de las variables calculables para la columna destiladora con recirculación

\begin{tabular}{|l|c|c||}
\hline \multicolumn{3}{|c|}{ VARIABLES CALCULABLES } \\
\hline \multicolumn{1}{|c|}{ Variable } & Valor & Unidades \\
\hline Flujo de flemas & 7535,5 & $\mathrm{~kg} / \mathrm{h}$ \\
\hline Flujo de vinazas & 52261,4 & $\mathrm{~kg} / \mathrm{h}$ \\
\hline Flujo del vapor de calentamiento & 9373,6 & $\mathrm{~kg} / \mathrm{h}$ \\
\hline Eficiencia de separación & 93,8 & $\%$ \\
\hline
\end{tabular}

Fuente: elaboración propia

Tabla 6. Resultados de las variables calculables para la columna destiladora con rehervidor y recirculación

\begin{tabular}{||l|c|c||}
\hline \multicolumn{3}{|c||}{ VARIABLES CALCULABLES } \\
\hline \multicolumn{1}{|c|}{ Variable } & Valor & Unidades \\
\hline Flujo de flemas & 8543,1 & $\mathrm{~kg} / \mathrm{h}$ \\
\hline Flujo de vinazas & 41879,9 & $\mathrm{~kg} / \mathrm{h}$ \\
\hline Flujo del vapor de calentamiento & 11604,9 & $\mathrm{~kg} / \mathrm{h}$ \\
\hline Eficiencia de separación & 95,1 & $\%$ \\
\hline
\end{tabular}

Fuente: elaboración propia 
Al comparar los resultados se aprecia que la inclusión de la recirculación aumenta el flujo de flemas (33\%) y vinazas (14\%) en la columna destiladora. El aumento del flujo de vinaza es perjudicial por constituir un residual altamente agresivo por su bajo $\mathrm{pH}$ y alta demanda química de oxígeno. Actualmente se realizan investigaciones dirigidas al uso de estas vinazas para reducir su impacto ambiental. [10], [11], [12]. Por lo tanto, la eficiencia de separación aumenta en un 1,7\%.

Por otra parte, la inclusión del rehervidor aumenta en un $24 \%$ el consumo de vapor de calentamiento por el uso de vapor indirecto, pero este puede recuperarse en el sistema como condensado, que dada su calidad, se puede utilizar como agua de alimentación de calderas, disminuyendo así el consumo de agua cruda. Adicionalmente, se reduce en un $20 \%$ el flujo de vinazas, lo cual reduce el efecto negativo sobre el ambiente. Como se aprecia, la eficiencia de la separación aumenta con la inclusión del rehervidor en 1,3\%.

Estos resultados se compararon con los que ofrece HYSYS $尺$, como simulador profesional reconocido y se encontró que los valores arrojados por este simulador son similares a los obtenidos por los modelos matemáticos desarrollados, siendo el error relativo menor de un 5\%. Con estos errores relativos se puede llegar a la conclusión de que los modelos matemáticos obtenidos son confiables para la simulación de columnas de destilación.

Respecto con la validación de los algoritmos para el conteo de platos teóricos, la herramienta de programación utilizada fue Visual Basic®. Para ello, además de los flujos, las composiciones y la temperatura de cada una de las corrientes, es necesario que se disponga de información sobre la cantidad de platos reales que tiene la columna. Este algoritmo permite determinar el plato de alimentación óptimo así como el perfil de temperatura en la columna.

Las tablas 7 y 8 reflejan las composiciones de líquido y vapor en cada plato de la columna, así como su temperatura, por el método de McCabe-Thiele y por el de Ponchon-Savarit. Ambos casos corroboran que al fondo de la columna se encuentra la zona de mayores temperaturas, puesto que aquí es donde está en mayor proporción el componente más pesado (en este caso el agua), mientras que la zona de tope está enriquecida por el etanol que es el componente más volátil, se encontró en esta zona las temperaturas más bajas.

Tabla 7. Perfil de la columna destiladora sin recirculación obtenido a partir del método de McCabe-Thiele

\begin{tabular}{|c|c|c|c||}
\hline \multicolumn{4}{|c|}{ Perfiles de la columna } \\
\hline Etapas teóricas & $\mathrm{T}^{\circ} \mathrm{C}$ & $\mathrm{x}_{1(\mathrm{~mol})}$ & $\mathrm{y}_{1(\mathrm{~mol})}$ \\
\hline 1 & 99,2 & 0,0035 & 0,0109 \\
\hline 2 & 98,8 & 0,0085 & 0,0341 \\
\hline 3 & 98,0 & 0,0187 & 0,0814 \\
\hline 4 & 96,3 & 0,0373 & 0,1676 \\
\hline
\end{tabular}

Fuente: elaboración propia

Tabla 8 Perfil de la columna destiladora sin recirculación obtenido a partir del método de Ponchon-Savarit

\begin{tabular}{|c|c|c|c|}
\hline \multicolumn{4}{|c|}{ Perfiles de la columna } \\
\hline Etapas teóricas & $\mathrm{T}^{\circ} \mathrm{C}$ & $\mathrm{x}_{1(\mathrm{~mol})}$ & $\mathrm{y}_{1(\mathrm{~mol})}$ \\
\hline 1 & 99,2 & 0,0029 & 0,0280 \\
\hline 2 & 98,9 & 0,0073 & 0,0698 \\
\hline 3 & 98,5 & 0,0142 & 0,1287 \\
\hline 4 & 97,8 & 0,0243 & 0,2062 \\
\hline 5 & 96,8 & 0,0386 & 0,2976 \\
\hline
\end{tabular}

Fuente: elaboración propia 
En la Tabla 9 se refleja la cantidad de platos teóricos obtenidos por ambos métodos. Los resultados arrojados por el método de Ponchon-Savarit son más exactos que los obtenidos por el de McCabe-Thiele, puesto que este último solo utiliza en sus cálculos las relaciones de equilibrio y considera la igualdad de los calores latentes molares de cambio de fase del agua y el etanol en la columna. Por otra parte, en el plato de alimentación existe transferencia de calor sensible entre el líquido del plato y la alimentación que se ingresa subenfriada y la presencia de un calor integral de dilución producto de la mezcla, lo cual no se tiene en cuenta en el método de McCabe-Thiele.

Tabla 9. Platos teóricos de la columna destiladora por ambos métodos

\begin{tabular}{|c|c|c|c|c||}
\hline \multirow{2}{*}{ Variable } & \multicolumn{2}{|c|}{ McCabe - Thiele } & \multicolumn{2}{c|}{ Ponchon- Savarit } \\
\cline { 2 - 5 } & Con recirculación & Sin recirculación & Con recirculación & Sin recirculación \\
\hline Platos teóricos & 2,90 & 3,99 & 3,94 & 4,63 \\
\hline $\begin{array}{c}\text { Plato de alimentación } \\
\text { teórico }\end{array}$ & 3 & 4 & 4 & 5 \\
\hline Eficiencia global (\%) & 15,3 & 21,0 & 20,7 & 24,4 \\
\hline
\end{tabular}

Se aprecia además que el número de platos en la columna con recirculación es menor que el requerido cuando se trabaja sin recirculación. Esto se debe a que aumenta la cantidad de líquido y vapor dentro de la columna, lo que exige que esta tenga mayor diámetro y espaciado entre platos. Con el aumento de líquido, aumenta la razón de reflujo y, con ello, se elevan además los gastos relacionados con el consumo de vapor, energía eléctrica y agua de enfriamiento [13].

\section{Conclusiones y Recomendaciones}

Se desarrollaron y validaron los modelos matemáticos propuestos para representar la columna destiladora con y sin rehervidor usada en la producción de etanol. Los resultados obtenidos muestran la confiabilidad de estos al ser los errores inferiores al 5\%, al establecer la comparación con HYSYS.

El método de Ponchon-Savarit es más riguroso y eficaz para la determinación de etapas teóricas y, por consiguiente, su eficiencia global, en una columna de destilación que el método de McCabe-Thiele; en consecuencia, su modelación es más compleja.

Se recomienda evaluar la sensibilidad del uso del método de determinación de la eficiencia global de la torre de destilación para la evaluación termoenergética de la instalación, para sustentar el uso del modelo más simple (McCabe-Thiele).

\section{Referencias Bibliográficas}

[1] Pérez, O., Gozá, O. Jimenez, O. (2003). Producción de alcohol por vía fermentativa. (Monogra- fía), CUJAE, Cuba. ISBN: 959-261-144-0.

[2] Silva, M A de Moraes. (2007). Es un contra- sentido decir que el etanol es una energía limpia. Brasil: Universidad de Sao Paulo.

[3] Nastari, Plinio M. (19-22 de junio, 2006) "El mercado mundial de etanol: visión global y perpectivas" [conferencia], IX Congreso Internacional sobre Azúcar y Derivados de la Caña, DIVERSIFICACIÓN 2006, Hotel Habana Libre Trip. 
[4] Macedo, Isaias C. (2007). Situação atual e perspectivas do etanol: Estúdios Avançados. 21 (59) 157 165.

[5] Gozá, O, Pérez de Alejo, H., Ricjckaert, M. (2002). Use of simulation and expert systems to increa- se the energy efficiency in cane sugar factories Published at Developments in Chemical Engineering \& Mineral Processing. Curtin University of Technology, Australia, special issue on Practical Applications of Process Systems Engineering, Volume 10, Numbers 1 and 2.

[6] Pérez, O. (2011). Modelación, simulación y análisis con fines energéticos de destilerías de etanol hidratado. (Tesis en opción al grado científico de Doctor en Ciencias Técnicas), Facultad de Ingeniería Química, CUJAE. Ciudad Habana: Cuba.

[7] Ruddy, D.F and Watson, $\mathrm{CH}$ (1987). Strategy for process engineering. New York: Wiley Publications Inc.

[8] ISSCT (International Society of Sugar Cane Technologies) Congress, Veracruz, Mexico, March 711, Vol.27Treybal Robert E. (1995). Operaciones de transferencia de masa. (2da Ed.) Ciudad: McGrawHill.

[9] Otiniano, M (200\&). Cálculos en destilación continua para sistemas binarios uti- lizando hoja de cálculo Excel. Lima, Perú: Universidad Nacional mayor de San Marcos, Ciudad Universi- taria s/n.

[10] Del Real, J., Prieto, F., Santos, E., Román, A. \& Gordillo, A. (200/). Factibilidad de un sistema de digestión anaerobia en el trata miento de aguas de proceso de vinazas con microorganismos de rumiantes. Rev.Técnica. Ingeniería. Universidad. Zulia. Vol. 30, № 2, 128 - 135.

[11] Satyawali, Y., Balakrishnan, M. (2008). Wastewater treatment in mo- lasses-based alcohol distilleries for COD and color removal. A review Journal of Environmental Manage- ment. 86 (2008) 481-497

[12] Tejada, M.A.G. (1995). Use of vinasse for soil reclamation and its impact on elemental loads in verti- sol soil and\} groundwater. (conference) Proceedings of XXVII.

[13] De Camargo, C. et al.: (1990). Conservacao de energia na industria do acucar e do alcool. Manual de recomendaciones. IPT, Sao Paulo, Brasil: Instituto de Pesquisas Tecnologicas.

[14] Scenna, N.J.et al. (1999). Modelado, simulación y optimización de procesos-, Argentina: Edición de la Universidad Tecnológica Nacional, ISBN: 950-42-0022-2. 